\title{
Avaliação Nutricional de Pessoas Com Deficiência Praticantes de Natação
}

\section{Nutritional Assessment of Disabled Subjects Practitioners of Swimming}

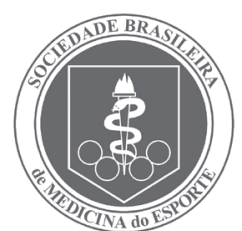

Artigo Original
Fabiana Simon Barreto

Cristina Panziera ${ }^{2}$

Marcelo Morganti Sant'Anna²

Marcello Ávila Mascarenhas ${ }^{3}$

Ana Paula Trussardi Fayh2,3

1. Acadêmica do curso de Nutrição - Centro Universitário Metodista IPA, Porto Alegre, RS.

2. Docente do Curso de Educação

Física - Centro Universitário

Metodista IPA, Porto Alegre, RS.

3. Docente do Curso de Nutrição

- Centro Universitário Metodista IPA,

Porto Alegre, RS.

\section{Endereço para correspondência:}

Ana Paula Trussardi Fayh

Rua Coronel Joaquim Pedro Salgado

80 - Rio Branco

90690-200 - Porto Alegre, RS, Brasil

Tels.: (51) 3316-1225/9342-8485. Fax:

(51) 3316-5811

E-mail: apfayh@yahoo.com.br

Submetido em 01/05/2008

Versão final recebida em 09/07/2008

Aceito em 28/11/2008

\begin{abstract}
RESUMO
A natação contribui para a saúde física, mental e social das pessoas que apresentam alguma deficiência. Sabendo que o aspecto nutricional é relevante para o desempenho esportivo e qualidade de vida desses indivíduos, o objetivo deste estudo foi avaliar o estado nutricional de pessoas com deficiências que praticam natação. Quatro indivíduos do sexo masculino e quatro do feminino, com deficiências físicas ou visuais, idade entre 19 e 36 anos, que nadavam de duas a três vezes por semana, participaram do protocolo de avaliação. Foram realizadas avaliação antropométrica, avaliação da ingestão alimentar através de recordatório de três dias e avaliação bioquímica para análise do perfil lipídico (triglicerídeos, colesterol total e frações). O IMC médio encontrado foi de $24,15 \pm 2,33 \mathrm{~kg} / \mathrm{m}^{2}$ e $27,26 \pm 4,91 \mathrm{~kg} / \mathrm{m}^{2}$ para homens e mulheres, respectivamente. O consumo alimentar médio encontrado, para ambos os sexos, foi de 3161,33 \pm 480,08kcal, tendo composição média de 58,13 \pm 5,04\% de carboidratos, 19,62 \pm 1,54\% de proteínas e $22,45 \pm 5,64 \%$ de lipídios do valor energético total (VET). As mulheres apresentaram consumo adequado de proteínas; entretanto os homens consumiam além das suas necessidades (2,14 \pm 0,34g/kg/dia). Para os demais nutrientes, o consumo foi adequado em ambos os sexos. As análises bioquímicas demonstraram, em geral, perfil lipídico adequado, com tendência a baixos valores de HDL. Conclui-se que os indivíduos do sexo masculino apresentaram composição corporal e perfil lipídico adequados, mesmo com consumo excedente de calorias e proteínas. Já no sexo feminino, verificou-se risco nutricional, pois as participantes apresentavam sobrepeso. Evidencia-se que esse grupo necessita de orientação nutricional individualizada para corrigir as inadequações alimentares observadas e, assim, melhorar seu desempenho físico e qualidade de vida.
\end{abstract}

Palavras-chave: avaliação nutricional, deficiência física, exercício.

\section{ABSTRACT}

Swimming contributes to physical, mental and social health of people who have some kind of disability. Bearing in mind that the nutritional aspect is relevant to sports performance as well as to life quality of these individuals, this study has aimed at evaluating the nutritional status of disabled individuals who practice swimming. Four male and four female individuals aged between 19 and 36 years who have either physical or visual impairment, as well as swim two or three weekly times, participated in the assessment protocol. Anthropometric analysis and food intake evaluation were used through three-day-recording and biochemical evaluation for the analysis of the lipid profile (triglycerides, total and fraction cholesterol). Mean BMl found was $24.15 \pm 2.33 \mathrm{~kg} / \mathrm{m} 2$ and $27.26 \pm 4.91 \mathrm{~kg} / \mathrm{m} 2$ for men and women, respectively. The average food intake observed for both sexes was $3161.33 \pm 480.08 \mathrm{kcal}$, having mean composition of $58.13 \pm 5.04 \%$ of carbohydrates, $19.62 \pm 1.54 \%$ of protein and $22.45 \pm 5.64 \%$ of lipids from the total energetic value (TEV). Women presented adequate intake of protein, while men ingested it beyond their needs $(2.14 \pm 0.34 \mathrm{~g} / \mathrm{kg} /$ day). Concerning the other nutrients, intake was adequate for both sexes. The biochemical analyses showed adequate lipid profile in general, with tendency for low HDL levels. It was concluded that the male individuals had satisfactory body composition and lipid profile, despite excessive intake of calories and proteins, while the female individuals presented nutritional risk due to overweight. It becomes evident the later group needs individualized nutritional orientation so as to correct their inadequate eating habits and hence improve their physical performance and life quality.

Keywords: nutritional assessment, physical disability, exercise. 


\section{INTRODUÇÃO}

A deficiência é definida como perda ou anormalidade de alguma estrutura ou função psicológica, fisiológica ou anatômica, que pode ser de origem congênita ou adquirida, permanente ou temporária ${ }^{(1)}$. A Constituição Federal Brasileira assegura diversos direitos a pessoas com deficiências, entre os quais se citam educação, cuidados com a saúde, assistência social, atendimento especializado, reabilitação, integração e participação à sociedade, proibição de discriminação e acessibilidade a instalações ${ }^{(2)}$. O esporte insere-se nesse contexto, pois se torna um instrumento para a integração do individuo à sociedade. A pessoa com deficiência que pratica alguma atividade física adquire independência, espírito competidor e melhoria nas relações com outras pessoas, que são fundamentais para a vida social. Tudo isso contribui para seu desenvolvimento pessoal e o exercício pleno de sua cidadania ${ }^{(3)}$.

A natação é considerada um dos esportes mais indicados para indivíduos com deficiência física, pois a água facilita a execução dos movimentos, além de contribuir para o desenvolvimento da coordenação, diminuição dos espasmos e promoção de relaxamento muscular ${ }^{(4)}$. Além de tudo, tanto para deficientes físicos como para visuais, a natação melhora o poder de concentração e a disciplina ${ }^{(3)}$.

A alimentação adequada é uma das chaves para o bom desempenho no exercício e para a saúde ${ }^{(5)}$. Os cuidados nutricionais em pessoas com deficiência devem ser redobrados, pois são mais suscetíveis a apresentar osteoporose ${ }^{(6)}$, cálculo renal, alteração do metabolismo dos carboidratos, proteínas e lipídios. Adicionalmente, têm maior probabilidade de desenvolver doenças cardiovasculares ${ }^{(7-9)}$. Amputados necessitam de aporte calórico maior, pois gastam mais energia para a execução dos movimentos devido à dificuldade de locomoção. 0 peso corporal deve ser controlado para não sobrecarregar o membro presente, evitando o desenvolvimento de problemas ósseos, pois o membro imobilizado está sujeito a descalcificações ${ }^{(10)}$.

Apesar do vasto conhecimento sobre a importância do exercício físico e da nutrição adequada na melhora da qualidade de vida, poucos estudos são encontrados na literatura avaliando o consumo alimentar de pessoas com deficiências engajadas em esportes. A caracterização alimentar dessa população é de suma importância, pois proporciona melhor compreensão de suas necessidades e facilita o desenvolvimento de intervenções nutricionais, com o objetivo de melhorar a qualidade de vida e o rendimento esportivo. Com isso, o objetivo deste estudo foi avaliar o estado nutricional de pessoas com deficiências físicas ou visuais que praticam natação em projeto paradesportivo.

\section{MÉTODOS}

\section{Amostra}

A amostra deste trabalho foi composta de oito participantes com deficiência, com idade entre 19 e 36 anos, participantes de projeto paradesportivo, o qual tem como objetivo incentivar a prática esportiva, o condicionamento e a manutenção física desses indivíduos. O grupo era formado por quatro pessoas do sexo masculino (três deficientes físicos e um visual) e quatro do sexo feminino (duas deficientes físicas e duas visuais). Um dos participantes do sexo masculino não possuía o membro inferior esquerdo abaixo do joelho e outro apresentava dismetria de membros inferiores, sendo o membro direito $5 \mathrm{~cm}$ mais longo do que o esquerdo. Todos os indivíduos praticavam natação de duas a três vezes por semana em piscina semiolímpica de um centro universitário. Este estudo foi aprovado pelo Comitê de Ética em Pesquisa da Rede Metodista de Educação do Sul (protocolo 61/2007). Todos os participantes foram elucidados sobre os procedimentos e objetivos da pesquisa e assinaram termo de consentimento livre e esclarecido em duas vias.

\section{Avaliação antropométrica}

Para a avaliação antropométrica, foram medidas a massa corporal, estatura, quatro dobras cutâneas e a circunferência da cintura. Todas as medidas foram obtidas antes do início do treinamento, os participantes utilizando roupas de banho. A massa corporal foi verificada com a balança digital Britânia ${ }^{\circledast}$, com capacidade máxima para 150kg. Devido ao fato de que um dos indivíduos não possuía o membro inferior esquerdo, realizou-se correção acrescentando-se $7 \%$ a seu peso ${ }^{(11)}$ para o cálculo do índice de massa corporal (IMC). A verificação da estatura foi realizada através do estadiômetro de parede da marca Cescorf ${ }^{\circledast}$, estando os participantes em posição ereta, com os braços estendidos ao longo do corpo e os pés descalços e unidos. Para minimizar erros de medida devido à falta de equilíbrio, ofereceu-se um suporte de apoio para o indivíduo amputado, bem como para o com dismetria de membros inferiores. O IMC foi obtido a partir da massa corporal (kg) dividida pela altura ao quadrado (metros), sendo utilizados os pontos de corte segundo a Organização Mundial da Saúde (OMS) ${ }^{(12)}$.

Foram medidas as seguintes dobras: tricipital (determinada paralelamente ao eixo longitudinal do braço, na face posterior, sendo seu ponto a distância média entre a borda súpero-lateral do acrômio e do olécrano), abdominal (determinada paralelamente ao eixo longitudinal do corpo, aproximadamente $2 \mathrm{~cm}$ à direita da borda lateral da cicatriz umbilical), subescapular (obtida obliquamente ao eixo longitudinal seguindo a orientação dos arcos costais, estando localizada $2 \mathrm{~cm}$ abaixo do ângulo inferior da escápula) e suprailíaca (realizada no sentido oblíquo, 2cm acima da crista ilíaca ântero-superior à altura da linha axilar média) ${ }^{(13)}$, utilizando-se o plicômetro clínico Cescorf. As medidas foram tomadas do lado direito do corpo, sendo realizadas três mensurações em cada local para a obtenção da média ${ }^{(13)}$. O percentual de gordura foi calculado através do protocolo de Faulkner ${ }^{(14)}$. Para a medida do perímetro da cintura, utilizou-se uma fita métrica inextensível e a medida foi tomada na altura média entre a última costela flutuante e o ponto ílio-cristal|(13). Para a classificação do risco cardiovascular pela circunferência da cintura, utilizaram-se os pontos de corte sugeridos pela OMS ${ }^{(12)}$.

\section{Avaliação dietética}

Para avaliar o consumo alimentar, foi utilizado um recordatório alimentar de três dias preenchido pelos próprios participantes ou por algum familiar próximo (no caso de deficiência visual), após a orientação do pesquisador. Os participantes foram instruídos a escolher dois dias da semana e um dia do final de semana para o registro de sua alimentação. Os alimentos foram expressos em medidas caseiras e todos os registros foram recolhidos e revisados pelo pesquisador responsável, juntamente com os avaliados, para o esclarecimento de dúvidas. As medidas caseiras foram convertidas em gramas e mililitros $^{(15)}$ para o cálculo do registro. Para a análise quantitativa de energia, macronutrientes (carboidratos, proteínas e lipídios), micronutrientes (vitamina C, sódio, cálcio, ferro), colesterol e fibra ingeridos foi utilizado o programa Dietwin Analise Nutricional". As informações obtidas foram comparadas com recomendações para indivíduos engajados em atividades físicas ${ }^{(5)}$ e com a ingestão dietética de referência (Dietary Reference Intakes - DRI) $)^{(16,17)}$.

\section{Avaliação bioquímica}

A avaliação bioquímica foi realizada em cinco voluntários que compareceram na data agendada, em período matutino, após jejum de 12 horas. Todas as coletas de amostras foram realizadas por técnicos do Laboratório de Toxicologia e Bioquímica do Centro Universitário Metodista IPA. Para a avaliação bioquímica esperou-se o soro coagular 
e centrifugou-se a 1.500rpm durante 10 minutos. Foi retirado apenas o sobrenadante (soro) para as análises e, em cada uma, foram utilizados kits da Labtest ${ }^{\oplus}$. A dosagem de triglicerídeos, colesterol total e HDL-C foi determinada pelo método enzimático colorimétrico e o LDL-C foi estimado pela fórmula de Friedwald(18).

\section{RESULTADOS}

A população deste estudo apresentou média de idade de 29,75 \pm 6,06 anos. Os valores médios antropométricos dessa população estão expressas na tabela 1. Ao analisarmos o IMC dos indivíduos, pode-se classificar o estado nutricional da amostra como sobrepeso. Entretanto, quando se estratifica a amostra por sexo, observa-se que apenas as mulheres se encontravam com sobrepeso. O percentual de gordura para ambos os sexos estava dentro da faixa da normalidade e o valor médio da circunferência da cintura só se apresentou aumentado para o sexo feminino.

Tabela 1. Dados antropométricos dos praticantes de natação.

\begin{tabular}{l|c|c|c}
\hline & $\begin{array}{c}\text { Homens } \\
(\mathbf{n}=\mathbf{4})\end{array}$ & $\begin{array}{c}\text { Mulheres } \\
(\mathbf{n}=\mathbf{4})\end{array}$ & $\begin{array}{c}\text { Total } \\
(\mathbf{n}=\mathbf{8})\end{array}$ \\
\hline Massa corporal (kg) & $62,67 \pm 5,70$ & $70,22 \pm 18,91$ & $66,45 \pm 13,54$ \\
\hline Estatura (cm) & $162,75 \pm 5,67$ & $157,50 \pm 9,03$ & $160,12 \pm 7,52$ \\
\hline IMC (kg/m²) & $24,15 \pm 2,33$ & $27,26 \pm 4,91$ & $25,70 \pm 3,93$ \\
\hline Dobra cutânea tríceps (mm) & $12,64 \pm 5,88$ & $22,38 \pm 7,10$ & $17,50 \pm 7,97$ \\
\hline Dobra cutânea subescapular (mm) & $15,47 \pm 3,93$ & $24,90 \pm 11,31$ & $20,18 \pm 9,32$ \\
\hline Dobra cutânea suprailíaca (mm) & $16,78 \pm 7,84$ & $23,27 \pm 9,72$ & $20,02 \pm 8,88$ \\
\hline Dobra cutânea abdominal (mm) & $23,03 \pm 4,15$ & $31,18 \pm 8,28$ & $27,10 \pm 7,46$ \\
\hline Percentual de gordura (\%) & $17,47 \pm 1,91$ & $21,34 \pm 5,06$ & $19,40 \pm 4,10$ \\
\hline Peso gordo (kg) & $10,2 \pm 2,57$ & $15,69 \pm 7,33$ & $12,94 \pm 5,87$ \\
\hline Peso magro (kg) & $52,47 \pm 4,12$ & $78,79 \pm 55,33$ & $65,63 \pm 38,95$ \\
\hline Circunferência da cintura (cm) & $81,62 \pm 2,62$ & $88,12 \pm 16,63$ & $84,87 \pm 11,55$ \\
\hline
\end{tabular}

Os dados do consumo alimentar estão ilustrados na tabela 2. Os indivíduos do sexo masculino apresentaram consumo energético levemente acima das recomendações. O consumo de carboidratos encontrou-se adequado, tanto pela avaliação percentual do macronutriente no total energético quanto pelas necessidades deste por quilograma de peso corporal ( $\mathrm{g} / \mathrm{kg} / \mathrm{dia}$ ). Os homens possuem consumo proteico elevado, mas as mulheres tiveram seu consumo classificado como satisfatório. O consumo de lipídios dos participantes se encontrou adequada, bem como o consumo dos demais nutrientes.
Tabela 2. Dados do consumo alimentar dos praticantes de exercício, obtidos através de recordatório alimentar de três dias

\begin{tabular}{l|c|c|c}
\hline & $\begin{array}{c}\text { Homens } \\
(\mathbf{n}=\mathbf{4})\end{array}$ & $\begin{array}{c}\text { Mulheres } \\
\mathbf{( n = 4 )}\end{array}$ & $\begin{array}{c}\text { Total } \\
\mathbf{( n = 8 )}\end{array}$ \\
\hline VET (kcal) & $3.410,51 \pm 340,52$ & $2.912,15 \pm 506,23$ & $3.161,33 \pm 480,08$ \\
\hline Kcal/kg & $54,54 \pm 4,97$ & $42,42 \pm 4,82$ & $48,48 \pm 7,90$ \\
\hline Carboidratos (\%) & $57,81 \pm 6,49$ & $58,46 \pm 4,12$ & $58,13 \pm 5,04$ \\
\hline Carboidratos (g/kg/dia) & $6,20 \pm 1,20$ & $5,01 \pm 1,04$ & $5,60 \pm 1,22$ \\
\hline Proteína (\%) & $20,14 \pm 1,72$ & $19,1 \pm 1,35$ & $19,62 \pm 1,54$ \\
\hline Proteína (g/kg/dia) & $2,14 \pm 0,34$ & $1,56 \pm 0,17$ & $1,85 \pm 0,39$ \\
\hline Lipídeo (\%) & $22,03 \pm 8,13$ & $22,87 \pm 2,77$ & $22,45 \pm 5,64$ \\
\hline Lipídeo (g/kg/dia) & $2,32 \pm 0,79$ & $1,91 \pm 0,25$ & $2,11 \pm 0,58$ \\
\hline Fibras (g) & $31,5 \pm 8,68$ & $26,40 \pm 3,03$ & $28,95 \pm 6,61$ \\
\hline Vitamina C (mg) & $213,77 \pm 145,75$ & $129,06 \pm 51,64$ & $171,42 \pm 110,89$ \\
\hline Cálcio (mg) & $998,21 \pm 152,05$ & $859,39 \pm 236,21$ & $928,80 \pm 198,31$ \\
\hline Ferro (mg) & $26,42 \pm 6,87$ & $20,69 \pm 3,70$ & $23,55 \pm 5,96$ \\
\hline Sódio (mg) & $2.299,89 \pm 1.693,34$ & $2.036,64 \pm 799,28$ & $2.168,26 \pm 1.233,89$ \\
\hline Colesterol (mg) & $322,76 \pm 112,01$ & $239,4 \pm 52,54$ & $281,08 \pm 92,44$ \\
\hline
\end{tabular}

O perfil lipídico dos participantes está ilustrado na tabela 3. Devido ao fato de apenas cinco participantes terem realizado a coleta de sangue, optou-se por expressar seus dados individualmente. Percebe-se adequação do colesterol total entre os participantes e tendência a baixos valores de colesterol HDL. Em relação aos triglicerídeos, duas pessoas apresentaram valores superiores aos das recomendações para prevenção de aterosclerose.

\section{DISCUSSÃO}

São escassos os trabalhos sobre avaliação nutricional de pessoas com deficiências físicas ou visuais. A inexistência de recomendações alimentares e de protocolos para avaliação antropométrica específicos para essa população dificulta a análise dos dados encontrados. Com isso, este estudo limita-se a descrever a amostra estudada, não tendo o poder de extrapolar os dados para a população. Deve-se apontar que as possíveis diferenças entre as necessidades especiais apresentadas pelos indivíduos também limita a discussão dos dados, bem como o pequeno tamanho amostral. A caracterização alimentar dessa população é fundamental, pois auxilia no desenvolvimento de métodos apropriados de intervenção nutricional, para melhorar a qualidade de vida, evitando o aparecimento de doenças crônicas não transmissíveis.

Tabela 3. Dados bioquímicos dos praticantes de exercício

\begin{tabular}{l|c|c|c|c|c|c}
\hline & \multicolumn{3}{|c|}{ Homens } & \multicolumn{2}{c}{ Mulheres } & \multirow{2}{*}{ Valores de referência* } \\
\hline & IND1 & IND2 & IND3 & IND1 & IND2 \\
\hline Colesterol total $(\mathrm{mg} / \mathrm{dl})$ & 136,4 & 147,7 & 186,4 & 161,7 & 122,1 & $<200$ \\
\hline $\mathrm{HDL}$ colesterol $(\mathrm{mg} / \mathrm{dl})$ & 32,0 & 39,4 & 51,0 & 55,1 & 34,0 & $\geq 40 \geq 50 * *$ \\
\hline LDL colesterol $(\mathrm{mg} / \mathrm{dl})$ & 85,0 & 91,7 & 101,39 & 72,98 & 108,1 & 100,0 \\
\hline Triglicerídeos $(\mathrm{mg} / \mathrm{dl})$ & 97,0 & 83,0 & 170,0 & 168,1 & $<150$ \\
\hline
\end{tabular}

* IV Diretriz Brasileira Sobre Dislipidemias e Prevenção da Aterosclerose ${ }^{(49)}$

${ }^{* *} \geq 40$ para homens e $\geq 50$ para mulheres ${ }^{(49)}$

IND - indivíduo 
Em relação aos valores do IMC, percebeu-se que os indivíduos do sexo masculino tiveram seu estado nutricional classificado como eutrófico ${ }^{(12)}$. Dados semelhantes foram encontrados para homens com deficiências físicas ou visuais engajados em esportes como futebol, natação ou basquete ${ }^{(19-21)}$. Na população feminina do presente estudo, o sobrepeso foi predominante, pois três participantes estavam acima do peso. Já Guimarães e Santos ${ }^{(20)}$ encontraram diferentes dados ao avaliar a composição corporal dos atletas brasileiros participantes das Paraolimpíadas de Sydney em diferentes esportes. Na natação feminina, composta de três atletas com paraplegia ou deficiência visual com média de idade de 26,57 \pm 7,50 anos, observou-se IMC médio de $20,11 \pm 1,14 \mathrm{~kg} / \mathrm{m}^{2}$. Vale ressaltar que o estudo citado foi realizado com atletas e nossa amostra era de praticantes de exercícios com finalidades não competitivas.

Com relação à adiposidade, as mulheres apresentaram valores superiores que os homens em todas as dobras cutâneas. O percentual de gordura dos indivíduos do presente estudo se encontrou dentro dos limites aceitos para a população em geral para ambos os sexos ${ }^{(22)}$. Já quando comparamos os valores de adiposidade com as recomendações para indivíduos engajados em treino de natação(22), os homens tiveram sua adiposidade classificada como acima da normalidade. Entretanto, esse valor recomendado para nadadores pode não ser um bom parâmetro para a comparação dos resultados da amostra estudada, pois não diferencia os valores de atletas e praticantes de exercício. Atletas possuem maior volume e intensidade de treinamento e isso resulta em modificações mais acentuadas em sua composição corporal(23). Em geral, os valores de circunferência da cintura se encontraram acima dos pontos de corte da OMS no sexo feminino ${ }^{(12)}$. Guimarães e Santos ${ }^{(20)}$ somaram os valores das circunferências das regiões centrais (cintura, peito e abdome) e os das regiões periféricas (quadril, coxa e panturrilha) de um grupo de três atletas paraolímpicas da natação para caracterização da distribuição de gordura dessa amostra. Duas atletas tiveram somatório maior nas regiões periféricas, apresentando composição corporal ginóide, que se relaciona com menor risco para o desenvolvimento de doenças cardiovasculares.

Em 2003, a Sociedade Brasileira de Medicina do Esporte ${ }^{(5)}$ publicou seu posicionamento oficial sobre as recomendações nutricionais para pessoas engajadas em atividades físicas, sendo estas atletas ou não. Os homens apresentaram consumo energético um pouco acima das recomendações, já as mulheres se encontraram dentro da recomendação. Esse consumo calórico considerado adequado no sexo feminino, mesmo com a identificação de sobrepeso e obesidade, pode ser devido a prévia modificação em seus hábitos alimentares, devido à identificação desse sobrepeso no início da prática de exercícios. Com isso, a necessidade da perda de peso para melhorar o desempenho durante os treinos pode ter ocasionado a diminuição de seu consumo energético mesmo sem orientação profissional. Para o indivíduo com amputação, não se pode afirmar que esse aporte calórico esteja adequado. Acredita-se que juntamente com a prática esportiva o aporte calórico aumente ${ }^{(19)}$.

Todos os participantes do estudo apresentaram ingestão adequada de carboidratos e lipídios. Os estudos disponíveis na literatura que avaliaram o consumo de macronutrientes de nadadores apresentam resultados contraditórios. Em alguns, pode-se observar consumo adequado de todos os macronutrientes (carboidratos, proteínas e lipídios)(24,25); já em outros, os nadadores apresentaram baixo consumo de carboidratos e alta ingestão de proteínas ${ }^{(26,27)}$. Uma semelhança foi observada no estudo de Soares et al. ${ }^{(19)}$, que caracterizaram o perfil nutricional de quatro homens amputados jogadores de futebol da Seleção Brasileira, com idade média de 29,3 $\pm 5,6$ anos. Verificou-se consumo de carboidratos de 48 a 54\%, lipídios de 25 a 30\% do aporte calórico total, entretanto, consumo proteico acima das recomendações, com média de $3 \mathrm{~g} / \mathrm{kg} / \mathrm{dia}$. Silva et al. ${ }^{(21)}$ realizaram avaliação nutricional de paraplégicos praticantes de natação ou basquete e de sedentários. Os 16 homens que praticavam exercício apresentaram distribuição dos macronutrientes desbalanceada, com baixo percentual de carboidratos e elevado consumo proteico. Outros estudos reportam alto consumo de proteínas por homens com idade entre 15 e 55 anos, nadadores competitivos ou por praticantes de natação ${ }^{(28,29,)}$. Esse consumo acima das recomendações não determina o ganho de massa muscular e nem o aumento do desempenho. Adicionalmente, esse excesso de proteínas na alimentação pode sobrecarregar a função renal e aumentar a adiposidade ${ }^{(30)}$. Todos os participantes do presente estudo ingeriram quantidades adequadas de cálcio, ferro, vitamina C, sódio, colesterol e fibras. No estudo de Soares et al. ${ }^{(19)}$, já citado, observouse que dentre um grupo de quatro homens amputados jogadores de futebol, a metade não tinha consumo adequado de fibras, de vitamina $\mathrm{C}$ e de cálcio.

A identificação do perfil lipídico auxilia na identificação do risco cardiovascular, o que pode interferir no estado nutricional do indivíduo e em seu desempenho esportivo. Soares et al. ${ }^{(19)}$, com o intuito de caracterizar o perfil nutricional de jogadores amputados de futebol, também verificaram valores adequados de colesterol total, HDL-C, LDL-C e triglicerídios nos quatro atletas avaliados, resultados igualmente encontrados no estudo de Silva et al. ${ }^{(21)}$, que avaliaram 16 praticantes de atividades físicas paraplégicos. Paschoal e Amâncio(28), ao estudar oito nadadores do sexo masculino, encontraram valores satisfatórios de colesterol total, HDL-C e LDL-C. É conhecido que o treinamento contribui para a adequação dos níveis de colesterol e triglicerídeos sanguíneos ${ }^{(18)}$. Entretanto, a dificuldade de mobilização de pessoas com deficiência, aliada à diminuição da atividade física e estresse psicológico, poderia contribuir para um perfil lipídico aterogênico nessa população ${ }^{(19)}$. Talvez o volume de treino dos participantes do presente estudo ainda não tenha sido estímulo suficiente para adequar seus valores de HDL-C.

O presente estudo descreveu o estado nutricional de pessoas com deficiência praticantes de natação. Quando avaliamos a amostra sem estratificar por sexo, observou-se que os indivíduos, em média, apresentavam composição corporal classificada como sobrepeso, com consumo energético dentro das recomendações para esportistas, mesmo com um consumo excessivo de proteínas. Adicionalmente, apresentavam perfil lipídico dentro dos valores recomendados. Entretanto, quando estratificamos a amostra pelo sexo, percebe-se que o sobrepeso foi verificado nas participantes femininos, sendo os homens considerados eutróficos. O consumo energético esteve acima das recomendações para o sexo masculino, bem como o consumo proteico. Já no sexo feminino, o consumo alimentar foi considerado adequado. Ambos os sexos tiveram seu consumo de micronutrientes, fibras e colesterol considerado adequado. Ainda, as mulheres apresentaram perfil lipídico menos aterogênico, com valores de LDL-C inferiores aos dos homens. Com isso, percebe-se a necessidade de orientação nutricional individualizada, com o objetivo de melhorar sua qualidade de vida e aumentar o rendimento esportivo.

Todos os autores declararam não haver qualquer potencial conflito de interesses referente a este artigo. 


\section{REFERÊNCIAS BIBLIOGRÁFICAS}

1. World Health Organization (WHO). International classification of impairments, disabilities, and handicaps: a manual of classification relating to the consequences of disease. Geneva: World Health Organization, 1980. 207p.

2. BRASIL. Constituição da República Federativa do Brasil. Brasília: Senado Federal, 1988. [updated 2007 Out 14; cited 1988]. Disponível: http://www.portaldoservidor.pa.gov.br/pdf/Con 1988br.pdf/.

3. Gabbai AA, Oliveira ASB, Cunha MCB, Labronici RHDD. Esporte como fator de integração do deficiente físico na sociedade. Arq Neur-Psiq 2000;58:1092-9.

4. Chatard JC, Lavoie JM, Ottoz H, Randaxhe P, Cazorla G, Lacour JR. Physiological aspects of swimming performance for persons with disabilities. Med Sci Sports Exerc 1992;24:1276-82.

5. Sociedade Brasileira de Medicina do Esporte (SBME). Modificações dietéticas, reposição hídrica, suplementos alimentares e drogas: comprovação de ações ergogênicas e potenciais riscos para a saúde. Rev Bras Med Esporte 2003;9:43-56.

6. Carvalho DCL, Carvalho MM, Cliquet AJR. Osteoporose por desuso: aplicação na reabilitação do lesado medular. Acta Ortop Bras 2001;9:1-9.

7. Spungen AM, Bauman WA, Wang JW, Pierson RN. The relation between total body potassium and resting energy expenditure in individuals with paraplegia. Arch Phys Med Rehab 1993;74:965-8.

8. Bauman WA, Sungen AM, Rothstein J, Raza M, Rothstein J, Zhang RL, et al. Coronary artery disease: metabolic risk factors and latent disease in individuals with paraplegia. Mt Sinai J Med 1992;59:63-168

9. Kocina P. Body composition of spinal cord injured adults. Sports Med 1997;23:48-60.

10. Demirel S, Demirel G, Tukek T, Erk O, Yilmaz H. Risk factors for coronary heart disease in patients with spinal cord injury in Turkey. Spin Cord 2001;39:134-8.

11. Martins C. Protocolo de procedimentos nutricionais In: Manual de nutrição clínica: Hospital de Clínicas Porto Alegre. Porto Alegre: HCPA, 2005. 173p.

12. Organização Mundial da Saúde (OMS). Prevenindo e controlando a epidemia global. Geneva: Roca, 2004. 256p.

13. Filho JF. A prática da avaliação física. Testes, Medidas e Avaliação Física em Escolares, Atletas e Academias de Ginástica. 2aed. Rio de Janeiro: Shape, 2003. 266p

14. Faulkner JA. Physiology, swimming and diving, In: Falls H. Exercise Physiology. Baltimore: Academic Press, 1968. p. 415-46.

15. Pinheiro ABV, Lacerda EMA, Gomes MCS, Benzecry EH, Costa VM. Tabela para avaliação de consumo alimentar em medidas caseiras. 5a ed. São Paulo: 2005. 152p.

16. Food and Nutrition Board/Institute of medicine (FNB). Dietary reference intakes for energy, carbo- hydrate, fiber, fat, fatty acids, cholesterol, protein, and amino acids. Washington DC: National Academy Press, 2005. 1357p.

17. Padovani RM, Amaya-Farfán J, Colugnati FAB, Domene SMA. Dietary reference intakes: aplicabilidade das tabelas em estudos nutricionais. Rev Nut 2006;19:741-60

18. Sociedade Brasileira de Cardiologia. IV Diretriz Brasileira sobre dislipidemias e prevenção da aterosclerose - Departamento de Aterosclerose da Sociedade Brasileira de Cardiologia. Arq Bras Cardiol 2007;88:2-19.

19. Soares EA, Ribeiro BG, Gomes AIS. Caracterização nutricional de jogadores de elite de futebol de amputados. Rev Bras Med Esporte 2005;11:11-6

20. Guimarães FJSP, Santos SS. Avaliação antropométrica e de composição corporal de atletas paraolímpicos brasileiros. Rev Bras Med Esporte 2002;8:84-91.

21. Silva RC, Tirapegui J, Ribeiro SML, Pires ISO. Estudo controlado da influência da atividade física em fatores de risco para doenças crônicas em indivíduos lesados medulares paraplégicos do sexo masculino. Rev Bras Educ Fís Esp 2004;18:169-77.

22. Pitanga FJG. Testes, medidas e avaliações em educação física e esportes. 4a. ed. São Paulo: Phorte, 2005. 200p.

23. Azevedo LF, Brum PC, Rosemblatt D, Perlingeiro PS, Barretto ACP, Negrão CE, et al. Características cardíacas e metabólicas de corredores de longa distância do ambulatório de cardiologia do esporte e exercício, de um hospital terciário. Arq Bras de Cardiol 2007:88:1-21.

24. Lukaski HC, Hoverson BS, Gallagher SK, Bolonchuk WW. Physical training and copper, iron, and zinc status of swimmers. Am J Clin Nutr 1990;51:1093-9.

25. Williams MM, Hawley JA. Dietary intakes of age-grup swimmers. Brit J Sports Med 1991;25:154-8.

26. Kapazi IM, Ramos LAZ. Hábitos e consumo alimentares de atletas nadadores. Rev Nutr 1998;11:117-24.

27. Soares EA, Ishi M, Burini RC. Estudo antropométrico e dietético de nadadores competitivos de áreas metropolitanas da região sudeste do Brasil. Rev Saúde Pública 1994;28:9-19.

28. Paschoal VCP, Amancio OMS. Nutrition status of Brazilian elite swimmers. Int J Sport Nutr Exerc Metabol 2004;14:81-94.

29. Pamplona AP, Kapazi IAM. Avaliação dietética de praticantes de atividade física em diferentes modalidades: Estudo comparativo. Rev Nutr Pauta 2004;66:61-5.

30. Lemon PW. Effects of exercise on dietary protein requirements. Int J Sport Nutr Exerc Metabol 1998:8:426-47 